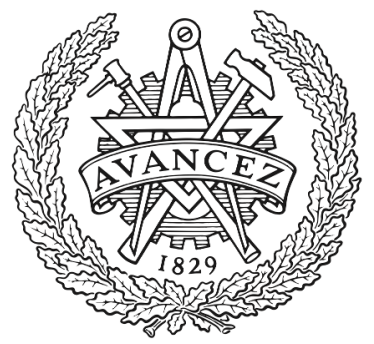

CHALMERS

UNIVERSITY OF TECHNOLOGY

\title{
A Nonreflecting Formulation for Turbomachinery Boundaries and Blade Row Interfaces
}

Downloaded from: https://research.chalmers.se, 2023-04-26 15:12 UTC

Citation for the original published paper (version of record):

Lindblad, D., Montero Villar, G., Andersson, N. et al (2019). A Nonreflecting Formulation for Turbomachinery Boundaries and Blade Row Interfaces. AIAA Scitech 2019 Forum. http://dx.doi.org/10.2514/6.2019-1804

N.B. When citing this work, cite the original published paper. 


\title{
A Nonreflecting Formulation for Turbomachinery Boundaries and Blade Row Interfaces
}

\author{
Daniel Lindblad *, Gonzalo Montero Villar ${ }^{\dagger}$, and Niklas Andersson ${ }^{\dagger}$ \\ Chalmers University of Technology, Gothenburg, SE-412 96, Sweden \\ Nathan A. Wukie ${ }^{\S}$ \\ University of Cincinnati, Cincinnati, Ohio, 45221
}

\begin{abstract}
Applying a nonreflecting formulation of a boundary condition or blade row interface is sometimes of paramount importance for obtaining an accurate prediction of turbomachinery blade flutter or tonal noise, just to name a few examples. Although the theoretical foundations for these type of boundary conditions have existed for several decades, nonreflecting boundary conditions still remain an area of active research. Today, much focus appears to be put towards obtaining more generic, higher-order and numerically stable formulations. In this work, a quasi-three-dimensional nonreflecting formulation based on the exact, nonreflecting boundary condition for a single frequency and azimuthal wave number developed by Giles is presented. The proposed formulation is applicable without modifications to both steady and unsteady simulations. An implementation strategy which is consistent for both a boundary condition and blade row interface is also presented. This implementation strategy does also partly address the stability problems often encountered when the type of formulation considered in the presented work is used together with a pseudo-time integration approach for converging the flow residual. Results from a set of two-dimensional validation cases are also presented to verify the formulation.
\end{abstract}

\section{Roman/Greek}

\section{Nomenclature}

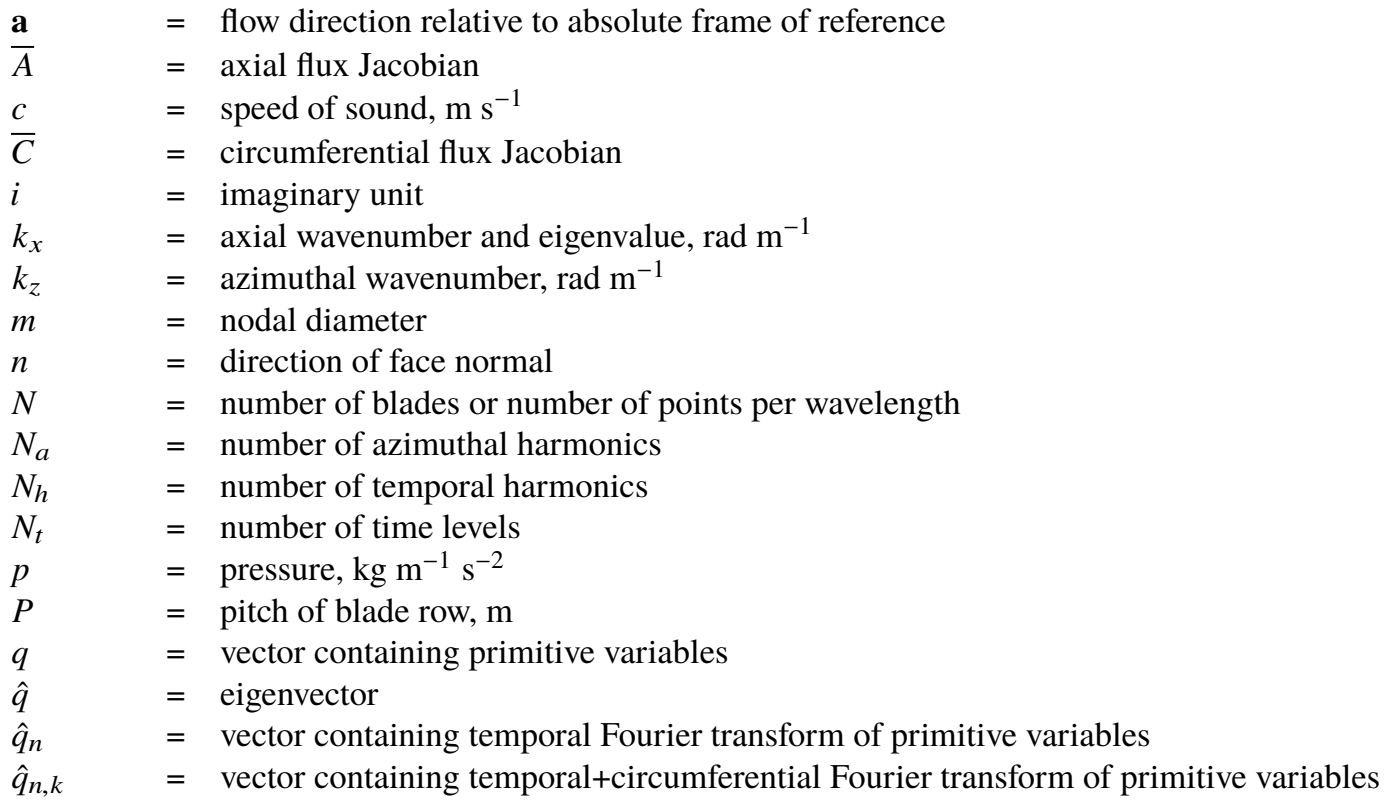

\footnotetext{
*Ph.D. Student, Department of Mechanics and Maritime Sciences, Hörsalsvägen 7A, SE-412 96 Gothenburg.

${ }^{\dagger}$ Ph.D. Student, Department of Mechanics and Maritime Sciences, Hörsalsvägen 7A, SE-412 96 Gothenburg.

† Assistant Professor, Department of Mechanics and Maritime Sciences, Hörsalsvägen 7A, SE-412 96 Gothenburg.

${ }^{\S}$ Ph.D. Student, Department of Aerospace Engineering, ML 70, Cincinnati, Ohio 45221, AIAA Student Member.
} 


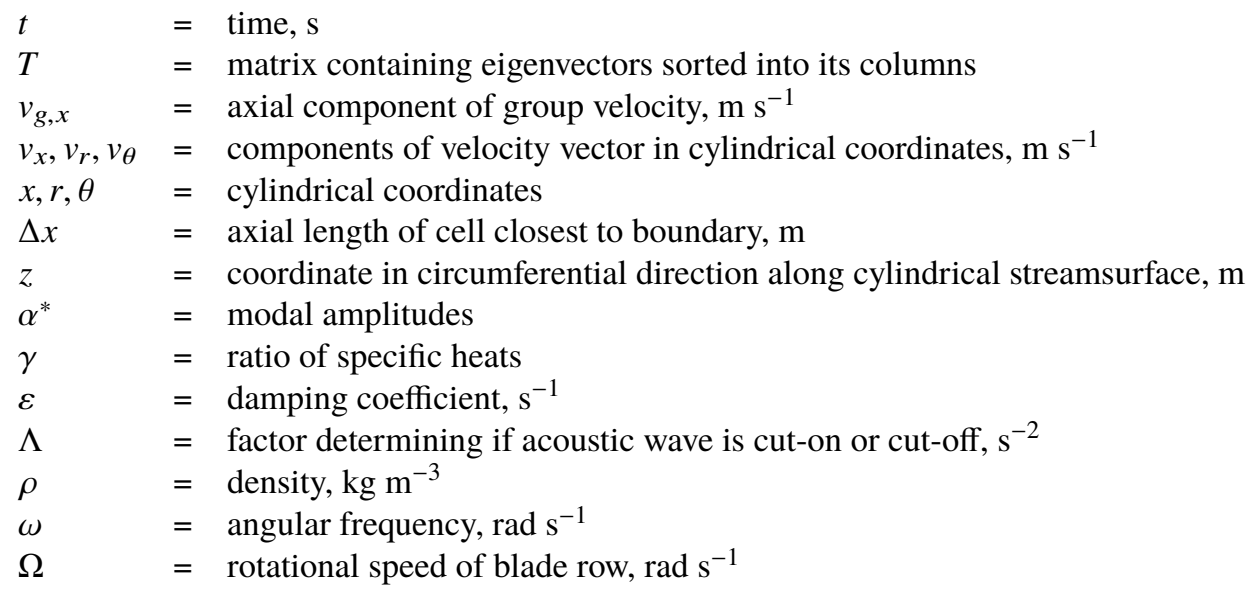

\section{Subscripts/Superscripts}

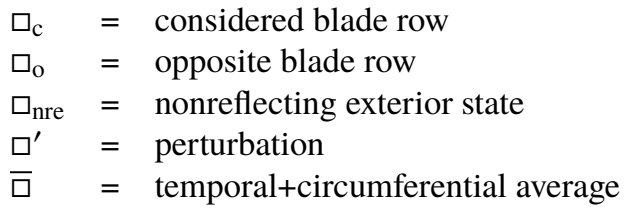

\section{Introduction}

UMERICAL simulations based on the Reynolds-averaged Navier-Stokes equations are today widely used to model the unsteady, three-dimensional flow field that is generated within an axial-flow turbomachine. These simulations can become very computationally expensive, especially when unsteady effects are considered. In order to resolve this issue, the computational domain is often set up to only include some parts the turbomachine. This approach often results in the domain boundaries ending up close to the turbomachinery blades, where the flow field can be highly non-uniform. In order to prevent these non-uniformities from degrading the solution quality, nonreflecting boundary conditions are often employed [1-5].

Early work on nonreflecting boundary conditions for hyperbolic problems was performed by Engquist and Majda [6]. An important result of their work is that a boundary condition only can be perfectly non-reflective if it is non-local in both space and time. Later, Giles [1,7] developed a set of nonreflecting boundary conditions for the linearized Euler equations. In this paper, the exact, nonreflecting boundary condition for a single frequency and azimuthal wave number developed by Giles has been adopted. This boundary condition is nonlocal in both space and time, and will thereby be exact within the linearized analysis. The adaption of Giles' boundary condition for a single frequency and azimuthal wave number presented in this work can be applied without modification for both steady and unsteady simulations, provided that a sampling of the flow in space and time can be computed. The regularization proposed by Frey et al. [8] has also been adapted within the current work to ensure that acoustic resonance is avoided.

It is well-known that a numerically stable implementation of the type of boundary condition considered in this work not is guaranteed when a pseudo-time integration approach is used to converge the residual [4, 7, 9]. In order to address this, a novel approach that lags the update of imposed boundary state is proposed. The implementation of this approach, together with the rest of the boundary condition, is explained in detail. It is also shown how the chosen nonreflecting formulation can be implemented consistently to both turbomachinery boundaries and to blade row interfaces. The latter application was previously considered by the present authors with the same nonreflecting formulation that is considered in this work [10]. The implementation is finally verified for a set of two-dimensional wave-propagation problems. 


\section{Method}

\section{A. Unsteady Blade Row Interaction}

The deterministic flow field within an axial turbomachine may be represented by a set of circumferential modes, each one having a certain nodal diameter and corresponding frequency with which it rotates. In this work, we restrict ourselves to cases when the turbomachine only consists of two blade rows in relative motion. For these cases, the modes will rotate relative to each blade row with the following frequencies [11]

$$
\omega_{n, \mathrm{c}}=n N_{\mathrm{o}}\left(\Omega_{\mathrm{o}}-\Omega_{\mathrm{c}}\right)
$$

Here, $N$ and $\Omega$ respectively denote the number of blades and rotational speed of a blade row, whereas the subscripts c and o indicate the blade row currently being considered and the opposite blade row respectively. Modes which rotate at the relative blade passing frequency defined in Eq. (1) are also restricted to the following nodal diameters [5, 12]

$$
m_{n, k, \mathrm{c}}=n N_{\mathrm{o}}+k N_{\mathrm{c}}
$$

The unsteady flow field relative to each blade row is obtained by summing up all the aforementioned modes in a double Fourier series expansion

$$
q_{\mathrm{c}}\left(x, r, \theta_{\mathrm{c}}, t\right)=\sum_{n=-N_{h}}^{n=N_{h}} \sum_{k=-N_{a}}^{N_{a}} \hat{q}_{n, k, \mathrm{c}}(x, r) e^{i\left(\omega_{n, \mathrm{c}} t-m_{n, k, \mathrm{c}} \theta_{\mathrm{c}}\right)}
$$

In this expression, $N_{h}$ and $N_{a}$ respectively denote the number of temporal and azimuthal harmonics that are included in the series expansion, and the vector $q=\left(\rho, v_{x}, v_{r}, v_{\theta}, p\right)^{T}$ is defined to contain the primitive solution variables in cylindrical coordinates and the absolute frame of reference. The combination of $q$ expressed in the absolute frame and the angle relative to the blade $\left(\theta_{\mathrm{c}}\right)$ in Eq. (3) is permissible, since the relative and absolute flow properties only differ in terms of the zeroth time-azimuthal Fourier coefficient $\left(\hat{q}_{0,0, c}\right)$. The variation of higher harmonics with respect to the absolute frame may be obtained by making the following substitution in Eq. (3)

$$
\theta_{\mathrm{c}}=\theta-\Omega_{\mathrm{c}} t
$$

If this is done one obtains the result that the relative blade passing frequency is Doppler shifted to the following frequency

$$
\omega_{n, k, \mathrm{c}}=n N_{\mathrm{o}} \Omega_{\mathrm{o}}+k N_{\mathrm{c}} \Omega_{\mathrm{c}}
$$

Together with the nodal diameter defined in Eq. (2), this frequency expresses how each mode rotates in the absolute frame of reference.

The nonreflecting boundary condition and blade row interface presented in this work are both constructed from time-azimuthal Fourier coefficients. These coefficients are calculated in two steps. In the first step, temporal Fourier coefficients of the primitive flow variables $\left(\hat{q}_{n, \mathrm{c}}\right)$ are calculated in all cells close to the boundary/interface. If the Harmonic Balance solver in G3D::Flow is employed to perform an unsteady simulation, the temporal Fourier coefficients are obtained from a discrete Fourier transform over the $N_{t}=2 N_{h}+1$ time levels that constitute the Harmonic Balance solution. The dual variable in the discrete Fourier transform should here be the relative blade passing frequency. If a steady state computation on the other hand is being performed, the only temporal Fourier coefficient which is of interest is the zeroth one, and its real part can thus be set equal to the solution itself. In the second step, the time-azimuthal Fourier coefficients are obtained from an azimuthal Fourier transform of the temporal Fourier coefficients as follows

$$
\hat{q}_{n, k, \mathrm{c}}(x, r)=\frac{N_{\mathrm{c}}}{2 \pi} \int_{0}^{2 \pi / N_{\mathrm{c}}} \hat{q}_{n, \mathrm{c}}\left(x, r, \theta_{\mathrm{c}}\right) e^{i m_{n, k, \mathrm{c}} \theta_{\mathrm{c}}} d \theta_{\mathrm{c}}
$$

Each time-azimuthal Fourier coefficient represents a flow perturbation (mode) with a known frequency and azimuthal wavenumber, c.f. Eq. (3). Each of these flow perturbations can in turn be split into upstream and downstream propagating waves based on the theory of Giles [1, 7] and Saxer and Giles [2]. The goal of a nonreflecting boundary condition is to drive the amplitude of all the incoming waves to zero. For a nonreflecting blade row interface on the other hand, the amplitude of each wave entering one side of the interface should match the amplitude of the corresponding wave 
exiting the other side of the interface [4]. The amplitude of the latter wave is obtained from the time-azimuthal Fourier coefficient sampled on the other side of the interface whose indices $l, p$ satisfy

$$
\begin{aligned}
& \omega_{n, k, \mathrm{c}}=\omega_{l, p, \mathrm{o}} \\
& m_{n, k, \mathrm{c}}=m_{l, p, \mathrm{o}}
\end{aligned}
$$

For the special case when the turbomachine consists of two blade rows which rotate relative to each other $\left(\Omega_{\mathrm{c}} \neq \Omega_{\mathrm{o}}\right)$, Eq. (7) can be solved to obtain the result that $l=k$ and $p=n$, or in other words, that $\hat{q}_{n, k, \mathrm{c}}$ corresponds to $\hat{q}_{k, n, \mathrm{o}}$. As a consequence of this, only modes which satisfy $n, k \leq \min \left(N_{h}, N_{a}\right)$ can be transferred across a blade row interface. When the Harmonic Balance method is used, this limit is often set by the number of temporal harmonics $\left(N_{h}\right)$ that are included in the computation. This is because the computational cost of the Harmonic Balance method scales (at best) linearly with the number of time levels, whereas a larger amount of azimuthal harmonics $\left(N_{a}\right)$ only requires more evaluations of the integral in Eq. (6), which typically is less expensive. An advantage of formulating the interface based on the nonreflecting theory of Giles [1, 7] and Saxer and Giles [2] is that all waves which do not have a counterpart in the corresponding blade row can still be treated with the nonreflecting analysis.

In the upcoming sections, the theory developed by Giles [1, 7] and Saxer and Giles [2] for decomposing modes into upstream and downstream propagating waves is presented. We then proceed to show how this theory can be used to formulate either a nonreflecting boundary condition, or a nonreflecting blade row interface which transmits waves that are resolved in each blade row. The final result will be a nonreflecting boundary condition/interface formulation which can be used for both steady state simulations, as well as unsteady simulations using the Harmonic Balance method.

\section{B. Linearized Euler Equations in a Thin Annular Duct}

The nonreflecting boundary conditions that were developed by Giles [1,7] apply to two-dimensional flows and are based on the assumption that the flow close to the boundary obeys the linearized Euler equations. When this theory is extended to three-dimensional flows inside an axial turbomachine, as was first done by Saxer and Giles [2], one instead assumes that the flow along a stream surface with constant radius obeys the following form of the linearized Euler equations

$$
\frac{\partial q^{\prime}}{\partial t}+\bar{A} \frac{\partial q^{\prime}}{\partial x}+\frac{1}{r} \bar{C} \frac{\partial q^{\prime}}{\partial \theta}=0
$$

In this equation, $q^{\prime}=q-\bar{q}$ represents a perturbation of the primitive flow variables away from a time-azimuthal average of the flow. The flux Jacobians in Eq. (8) are further defined in terms of this average as follows

$$
\bar{A}=\left[\begin{array}{ccccc}
\bar{v}_{x} & \bar{\rho} & 0 & 0 & 0 \\
0 & \bar{v}_{x} & 0 & 0 & 1 / \bar{\rho} \\
0 & 0 & \bar{v}_{x} & 0 & 0 \\
0 & 0 & 0 & \bar{v}_{x} & 0 \\
0 & \gamma \bar{p} & 0 & 0 & \bar{v}_{x}
\end{array}\right] \quad \bar{C}=\left[\begin{array}{ccccc}
\bar{v}_{\theta} & 0 & 0 & \bar{\rho} & 0 \\
0 & \bar{v}_{\theta} & 0 & 0 & 0 \\
0 & 0 & \bar{v}_{\theta} & 0 & 0 \\
0 & 0 & 0 & \bar{v}_{\theta} & 1 / \bar{\rho} \\
0 & 0 & 0 & \gamma \bar{p} & \bar{v}_{\theta}
\end{array}\right]
$$

Note that for a perfect ideal gas, $\gamma \bar{p}=\bar{\rho} \bar{c}^{2}$, where $\bar{c}$ is the average speed of sound and $\gamma$ the ratio of specific heats.

The accuracy of a nonreflecting boundary condition based on the linearized Euler equations will naturally decrease if the flow perturbations close to the boundary are large in amplitude, and/or if viscous effects are important. It can also be noted that the substitution $z=r \theta$ in Eq. (8) yields the linearized Euler equations in two dimensions. This shows that radial flow variations can not be completely accounted for when a nonreflecting boundary condition based on Eq. (8) is employed. More general nonreflecting boundary conditions than the one considered in this work can be constructed to account for radial variations [9, 13]. These will however require the whole boundary to be included simultaneously in the analysis, which can be compared with the current approach in which each radial span of the boundary can be considered independently. A nonreflecting boundary condition constructed based on Eq. (8) has also been shown by Saxer and Giles [2] to give better results than when the boundary condition is formulated based on local 1D characteristics.

\section{Modal Decomposition of the Linearized Euler Equations}

In this section the linearized Euler equations presented in Eq. (8) will be used to decompose a flow perturbation with a known frequency and azimuthal wave number into incoming and outgoing waves. In order to do this, one assumes that each of the waves vary harmonically in the axial direction and thereby can be written in the following form [1,7] 


$$
q^{\prime}(x, r, \theta, t)=\hat{q}(r) e^{i\left(\omega t-k_{x} x-k_{z} z\right)}
$$

Here, $k_{x}$ is the axial wavenumber of the wave and $k_{z} z=m \theta$, where $k_{z}=m / r$ is the azimuthal wavenumber of the wave and $z=r \theta$. The variable substitution $z=r \theta$ may also be applied in Eq. (8) to obtain

$$
\frac{\partial q^{\prime}}{\partial t}+\bar{A} \frac{\partial q^{\prime}}{\partial x}+\bar{C} \frac{\partial q^{\prime}}{\partial z}=0
$$

The linearity of Eq. (11) implies that each wave defined by Eq. (10) will propagate independently of the others. One can therefore proceed by substituting a single wave into Eq. (11) to obtain the following relation

$$
\left(\omega-k_{x} \bar{A}-k_{z} \bar{C}\right) \hat{q}(r)=0
$$

From this relation, an eigenvalue problem for the axial wavenumber, with corresponding eigenvector $\hat{q}(r)$, may be obtained by multiplying Eq. (12) from the left by $\bar{A}^{-1}$

$$
\left(\omega \bar{A}^{-1}-k_{z} \bar{A}^{-1} \bar{C}-k_{x} I\right) \hat{q}(r)=0
$$

This relation is only valid if $\bar{A}$ is invertible, which holds true if $\operatorname{det}(\bar{A})=\bar{v}_{x}^{3}\left(\bar{c}^{2}-\bar{v}_{x}^{2}\right)$ is nonzero. In order to ensure this, we will assume that the mean axial velocity is nonzero and subsonic. It can also be noted from Eq. (13) that $\left(\omega \bar{A}^{-1}-k_{z} \bar{A}^{-1} \bar{C}\right)$ only is nontrivial if $\omega$ and $k_{z}$ are not both zero at the same time. The special case when $\omega=k_{z}=0$ represents the mean flow and will not be treated by the present analysis. Instead, it will be used for prescribing mean flow boundary conditions or matching the mean flow at blade row interfaces, as will be described later.

The eigenvalue problem presented in Eq. (13) can be solved for a given combination of frequency and azimuthal wavenumber to obtain five eigenvalue/eigenvector pairs $\left(k_{x, j}, \hat{q}_{j}\right)$. These pairs may then be substituted back into Eq. (10) to obtain all the waves that are allowed to exist in the flow according to Eq. (11). Moreover, the axial wavenumbers obtained from the analysis can be used to compute the direction of propagation of the waves, whereas the structure of the eigenvectors will define the physical nature of the wave [7]. An eigenvector corresponding to a given eigenvalue is however not uniquely defined, since any multiplication by a scalar yields another eigenvector to the same eigenvalue. If the geometric multiplicity of a given eigenvalue is greater than one, then any linear combination of two eigenvectors corresponding to this eigenvalue will also become an eigenvector. As a result of this non-uniqueness, care must be taken when the eigenvectors are defined. In particular, it is advantageous to ensure that the eigenvectors have a physically relevant meaning in the sense that they correspond to entropy, vorticity and acoustic waves [7]. It is also important from a numerical point of view to ensure that the eigenvectors have been chosen so that a division by zero or a null vector never is obtained under any circumstance. The eigenvectors selected in this work satisfy all the aforementioned conditions. We start by presenting the eigenvalue/eigenvectors pairs corresponding to convected waves.

\section{Convected Waves}

The first eigenvalue of the matrix $\left(\omega \bar{A}^{-1}-k_{z} \bar{A}^{-1} \bar{C}\right)$ has an algebraic multiplicity equal to three and reads as follows

$$
k_{x, 1}=k_{x, 2}=k_{x, 3}=\frac{\omega-k_{z} \bar{v}_{\theta}}{\bar{v}_{x}}
$$

As it turns out, the geometric multiplicity of this eigenvalue is also three. In this work, the eigenvectors corresponding to the eigenvalue defined in Eq. (14) have been selected as shown below

$$
\hat{q}_{1}=\left[\begin{array}{c}
\bar{\rho} \\
0 \\
0 \\
0 \\
0
\end{array}\right] \quad \hat{q}_{2}=\left[\begin{array}{l}
0 \\
0 \\
\bar{c} \\
0 \\
0
\end{array}\right] \quad \hat{q}_{3}=\left[\begin{array}{c}
0 \\
-\bar{c} k_{z} \\
0 \\
\bar{c} k_{x, 1} \\
0
\end{array}\right]
$$

The structure of these eigenvectors shows that they respectively correspond to an entropy wave, radial vorticity wave and axial-circumferential vorticity wave. It can also be noted that under the stated assumptions, these vectors will always remain well defined in the sense that they never become a zero vector, nor include a division by zero. 
The direction of propagation of the waves defined by the first three eigenvalue/eigenvector pairs may be determined from the group velocity, since both the frequency and axial/circumferential wavenumbers are real-valued. The relevant component of the group velocity in our case is the axial one, which may be computed as [7]

$$
v_{g, x, j}=\frac{\partial \omega}{\partial k_{x, j}}=\left(\frac{\partial k_{x, j}}{\partial \omega}\right)^{-1}=\bar{v}_{x}, \quad j=1,2,3
$$

This result shows that the entropy and vorticity waves are convected downstream with the mean flow, as should be expected.

\section{Acoustic Waves}

The remaining two eigenvalues of $\left(\omega \bar{A}^{-1}-k_{z} \bar{A}^{-1} \bar{C}\right)$ may be written as follows

$$
\begin{aligned}
& k_{x, 4}=\frac{-\bar{v}_{x}\left(\omega-k_{z} \bar{v}_{\theta}\right)+\bar{c} \sqrt{\Lambda}}{\bar{c}^{2}-\bar{v}_{x}^{2}} \\
& k_{x, 5}=\frac{-\bar{v}_{x}\left(\omega-k_{z} \bar{v}_{\theta}\right)-\bar{c} \sqrt{\Lambda}}{\bar{c}^{2}-\bar{v}_{x}^{2}}
\end{aligned}
$$

where

$$
\Lambda=\left(\omega-k_{z} \bar{v}_{\theta}\right)^{2}-k_{z}^{2}\left(\bar{c}^{2}-\bar{v}_{x}^{2}\right)
$$

The eigenvectors corresponding to these eigenvalues have been selected as

$$
\hat{q}_{4}=\left[\begin{array}{c}
\bar{\rho} \\
\frac{-\bar{c}^{2} k_{x, 4}}{\bar{v}_{x} k_{x, 4}-\bar{v}_{x} k_{x, 1}} \\
0 \\
\frac{-\bar{c}^{2} k_{z}}{\overline{\bar{v}}_{x} k_{x, 4}-\bar{v}_{x} k_{x, 1}} \\
\bar{\rho} \bar{c}^{2}
\end{array}\right] \quad \hat{q}_{5}=\left[\begin{array}{c}
\bar{\rho} \\
\frac{-\bar{c}^{2} k_{x, 5}}{\bar{v}_{x} k_{x, 5}-\bar{v}_{x} k_{x, 1}} \\
0 \\
\frac{-\bar{c}^{2} k_{z}}{\bar{v}_{x} k_{x, 5}-\bar{v}_{x} k_{x, 1}} \\
\bar{\rho} \bar{c}^{2}
\end{array}\right]
$$

The structure of these eigenvectors shows that the last two eigenvalue/eigenvector pairs define two acoustic waves. In order to determine the direction of propagation of these waves we must first look to the factor $\Lambda$ defined in Eq. (18). If $\Lambda$ is strictly positive, the eigenvalues in Eq. (17) become real-valued and the group velocity can thus be used to determine the direction of propagation. The axial component of the group velocities for the acoustic waves becomes as follows

$$
\begin{aligned}
& v_{g, x, 4}=-\frac{\bar{c}^{2}-\bar{v}_{x}^{2}}{\bar{v}_{x}-\frac{\bar{c}\left(\omega-k_{z} \bar{v}_{\theta}\right)}{\sqrt{\Lambda}}} \\
& v_{g, x, 5}=-\frac{\bar{c}^{2}-\bar{v}_{x}^{2}}{\bar{v}_{x}+\frac{\bar{c}\left(\omega-k_{z} \bar{v}_{\theta}\right)}{\sqrt{\Lambda}}}
\end{aligned}
$$

If the mean axial velocity is subsonic and $\Lambda>0$ it is possible to show that if $v_{g, x, 4}>0$, then $v_{g, x, 5}<0$ and vice versa. Under these conditions, one acoustic wave will therefore propagate upstream and the other one downstream. If $\Lambda$ becomes strictly negative, one obtains two complex eigenvalues from Eq. (17), one with positive and one with negative imaginary part. If these eigenvalues are inserted into Eq. 10 it is found that a negative imaginary part corresponds to a wave that is exponentially decaying in the positive axial direction, whereas a positive imaginary part gives exponential decay in the negative axial direction. In this work, the direction of propagation of an acoustic wave with a complex axial wavenumber is taken to be the direction in which it decays. This definition has also been adopted by other authors [8, 14]. As a consequence of this definition, all acoustic waves associated with complex axial wavenumbers are interpreted as decaying from their respective point of origin, which motivates the commonly used abbreviation "cut-off". The acoustic waves associated with real wavenumbers will on the other hand propagate without decaying, and are therefore referred to as "cut-on".

It is important to note that the acoustic waves not have been ordered to ensure that the fourth and fifth one always propagate downstream and upstream respectively. Instead, the direction of propagation of these waves must be 
determined either from the group velocity or the axial wavenumber, dependent on whether the waves are cut-on or cut-off. More details on how the direction of propagation is accounted for will be presented later. Before this, however, the special case $\Lambda=0$ must be covered. This case is sometimes referred to as acoustic resonance [8] and yields two identical acoustic eigenvalue/eigenvector pairs as well as two axial group velocities that are zero. This is a problem since the construction of the nonreflecting boundary/interface presented in this work is dependent on the fact that there exist 5 linearly independent eigenvectors, for which the direction of propagation of the associated waves are known. In order to ensure that acoustic resonance does not occur, the regularization proposed by Frey et al. [8] has been adopted. This regularization will be outlined next.

\section{Regularization to Prevent Acoustic Resonance}

In order to prevent acoustic resonance solutions from existing, Frey et al. [8] propose a modification of the underlying linearized Euler equations to include a damping term according to

$$
\frac{\partial q^{\prime}}{\partial t}+\bar{A} \frac{\partial q^{\prime}}{\partial x}+\bar{C} \frac{\partial q^{\prime}}{\partial z}+\varepsilon q^{\prime}=0
$$

where $\varepsilon>0$ is a factor that sets the amount of damping. By substituting the harmonic wave assumption defined in Eq. (10) into Eq. 21, the following new eigenvalue problem may be obtained

$$
\left((\omega-i \varepsilon) \bar{A}^{-1}-k_{z} \bar{A}^{-1} \bar{C}-k_{x} I\right) \hat{q}(r)=0
$$

This eigenvalue problem can also be obtained by replacing $\omega$ in Eq. (13) with the following, modified frequency

$$
\tilde{\omega}=\omega-i \varepsilon
$$

As a result of this, the solution to the new eigenvalue problem in Eq. 22 may be obtained by replacing $\omega$ with $\tilde{\omega}$ in the solutions to Eq. (13). When this is done, it is found that the factor $\Lambda$ always becomes non-zero, thereby ensuring that the two acoustic eigenvalue/eigenvector pairs remain unique.

The modification of $\omega$ in Eq. (22) will also cause the two acoustic eigenvalues to be complex-valued, and thus always yield acoustic waves that are cut-off [8]. This raises a question regarding how the direction of propagation of an acoustic wave that is cut-on in the original analysis should be determined when the damping term is included. The answer is also provided by Frey et al. [8], who show that the direction of propagation of a wave that is cut-on in the original analysis, will be the same as the direction of damping of the wave when the modified frequency in Eq. 23. is used. This elegant result, which also has been verified numerically by the authors of this paper, allows us to treat all acoustic waves consistently when the modified frequency is being employed. That is, the direction of propagation of the acoustic waves can always be determined from the imaginary part of $k_{x, 4}, k_{x, 5}$ when the modification of of $\omega$ in Eq. 23. is employed. It should also be pointed out that the modification of $\omega$ only applies when the eigenvalues/eigenvectors are being computed, not for calculating the temporal evolution of the wave in Eq. 10]. The group velocity is also still used for computing the direction of propagation of the convected waves.

\section{Definition of Nonreflecting State at a Boundary or Interface}

The foregoing analysis has shown that five different waves exist for a given pair of frequency and azimuthal wavenumber which satisfy Eq. 11] (or Eq. 21] when acoustic resonance is accounted for). This suggests that the most general form of a flow perturbation with a given frequency and azimuthal wavenumber is a linear combination of all five waves

$$
q^{\prime}(x, r, \theta, t)=\sum_{j=1}^{5} \alpha_{j}(r) \hat{q}_{j}(r) e^{i\left(\omega t-k_{x, j} x-k_{z} z\right)}
$$

Here, $\alpha_{j}(r)$ is a complex number that defines the amplitude and phase of wave $j$. We can simplify the above equation slightly by absorbing the terms $e^{-i k_{x, j} x}$ into the corresponding amplitudes $\alpha_{j}(r)$ to obtain

$$
q^{\prime}(x, r, \theta, t)=\sum_{j=1}^{5} \alpha_{j}^{*}(x, r) \hat{q}_{j}(r) e^{i\left(\omega t-k_{z} z\right)}
$$


This equation can also be written in matrix notation as follows

$$
q^{\prime}(x, r, \theta, t)=T(r) \alpha^{*}(x, r) e^{i\left(\omega t-k_{z} z\right)}
$$

where $\alpha^{*}(x, r)$ is a $5 \times 1$ vector that contains the modal amplitudes $\alpha_{j}^{*}(x, r)$, and $T(r)$ is a $5 \times 5$ matrix that contains the eigenvectors $\hat{q}_{j}(r)$ structured into its columns

$$
T=\left[\begin{array}{ccccc}
\bar{\rho} & 0 & 0 & \bar{\rho} & \bar{\rho} \\
0 & 0 & -\bar{c} k_{z} & \frac{-\bar{c}^{2} k_{x, 4}}{\bar{v}_{x} k_{x, 4}-\bar{v}_{x} k_{x, 1}} & \frac{-\bar{c}^{2} k_{x, 5}}{\bar{v}_{x} k_{x, 5}-\bar{v}_{x} k_{x, 1}} \\
0 & \bar{c} & 0 & 0 & 0 \\
0 & 0 & \bar{c} k_{x, 1} & \overline{\bar{v}}_{x} k_{x, 4} \overline{\bar{v}}_{2} k_{x, 1} & \frac{-\bar{c}^{2} k_{z}}{\bar{v}_{x} k_{x, 5}-\bar{v}_{x} k_{x, 1}} \\
0 & 0 & 0 & \bar{\rho} \bar{c}^{2} & \bar{\rho} \bar{c}^{2}
\end{array}\right]
$$

From Eq. (3) it can now be noted that each time-azimuthal Fourier coefficient also represents a flow perturbation with a known frequency and nodal diameter. By rewriting one of these flow perturbations in the absolute frame of reference, for which the frequency is defined by Eq. (5), and then dropping the harmonic indices $n, k$ one obtains

$$
q^{\prime}(x, r, \theta, t)=\hat{q}_{c}(x, r) e^{i\left(\omega_{c} t-m_{c} \theta\right)}
$$

Under the previously stated assumptions the most general form of a flow perturbation with a known frequency and azimuthal wave number is given by Eq. 26). This suggests that if $k_{z}=m_{c} / r$ and $\omega=\omega_{c}$ in this equation, it may be equated with Eq. (28) to obtain

$$
\hat{q}_{c}(x, r)=T(r) \alpha^{*}(x, r)
$$

From this equation it can be seen that if $T(r)$ is invertible, then the amplitude and phase of the different waves can be measured as

$$
T^{-1}(r) \hat{q}_{c}(x, r)=\alpha^{*}(x, r)
$$

For a boundary, a nonreflecting state may now be defined as

$$
T_{\mathrm{inc}}^{-1}(r) \hat{q}_{c}(x, r)=0
$$

where $T_{\mathrm{inc}}^{-1}(r)$ is defined to contain the rows of $T^{-1}(r)$ corresponding to incoming waves. For an interface, on the other hand, the condition reads

$$
T_{\mathrm{inc}}^{-1}(r) \hat{q}_{c}(x, r)=T_{\mathrm{inc}}^{-1}(r) \hat{q}_{\mathrm{o}}(x, r)
$$

It should be noted that if Eq. (32) is satisfied on both sides of the interface, then it holds that $\hat{q}_{\mathrm{c}}=\hat{q}_{\mathrm{o}}$ [4]. Also note that the matrix $T_{\text {inc }}^{-1}(r)$ in Eqs. (31) and (32) is either of size $4 \times 5$ for an inlet, or $1 \times 5$ for an outlet. As such, these equations can not be used to define a unique $\hat{q}_{c}(x, r)$ for a given frequency, azimuthal wavenumber, mean flow state and possible exterior state. This is necessary from a physical point of view since the boundary only should specify information propagating into the domain. From a numerical point of view on the other hand, this complicates the construction of the boundary condition since one can find several ways to enforce Eqs. (31) and (32). As was noted by Giles [1], the chosen method can have a large impact on the convergence rate and stability of the solver. In this work, the amplitudes of the incoming waves are explicitly set to zero for a boundary, or to the value obtained on the other size of the interface. This approach has proven to give good accuracy, but can be unstable when an explicit pseudo-time integration strategy is used to converge the solution. More details on this will be presented in the next section. Before this is done, however, the inverse of $T(r)$ must be defined.

The inverse of $T(r)$ exists if it has full rank, which holds true if and only if the eigenvectors used to construct $T(r)$ are linearly independent. As noted previously, this is not true in the case of acoustic resonance. If it is assumed that acoustic resonance does not occur $(\Lambda \neq 0)$ and that the modification of $\omega$ in Eq. 23 is not employed, then it is a fairly simple task to prove that $T(r) \mathbf{x}=0$ only has trivial solutions under the previously stated assumptions. A similar proof for the case when $\omega$ is modified to avoid acoustic resonance has not been completed. It is however believed that $T(r)$ will be invertible in all circumstances when the modification of $\omega$ is used. Combined with the fact that care has been 
taken to ensure that the eigenvectors $\hat{q}_{j}(r)$ are always well-defined in the sense that they never become a zero vector or include a division by zero, this would then imply that the chosen formulation can be used without modification for a very wide range of problems. The inverse of $T(r)$ reads as follows

$$
T^{-1}=\left[\begin{array}{ccccc}
\overline{1} & 0 & 0 & 0 & \frac{-1}{\bar{\rho} \bar{c}^{2}} \\
0 & 0 & \frac{1}{\bar{c}} & 0 & 0 \\
0 & \frac{-k_{z}}{\bar{c}\left(k_{x, 1}^{2}+k_{z}^{2}\right)} & 0 & \frac{k_{x, 1}}{\bar{c}\left(k_{x, 1}^{2}+k_{z}^{2}\right)} & \overline{\bar{\rho} \bar{c} \bar{v}_{x}\left(k_{x, 1}^{2}+k_{z}^{2}\right)} \\
0 & \frac{-\bar{v}_{x}\left(k_{x, 4} k_{x, 1}-k_{x, 1}^{2}\right)}{2 \bar{c}^{2}\left(k_{x, 4} k_{x, 1}+k_{z}^{2}\right)} & 0 & \frac{-\bar{v}_{x}\left(k_{x, 4} k_{z}-k_{x, 1} k_{z}\right)}{2 \bar{c}^{2}\left(k_{x, 4} k_{x, 1}+k_{z}^{2}\right)} & \frac{1}{2 \bar{\rho} \bar{c}^{2}} \\
0 & \frac{-\bar{v}_{x}\left(k_{x, 5} k_{x, 1}-k_{x, 1}^{2}\right)}{2 \bar{c}^{2}\left(k_{x, 5} k_{x, 1}+k_{z}^{2}\right)} & 0 & \frac{-\bar{v}_{x}\left(k_{x, 5} k_{z}-k_{x, 1} k_{z}\right)}{2 \bar{c}^{2}\left(k_{x, 5} k_{x, 1}+k_{z}^{2}\right)} & \frac{1}{2 \bar{\rho} \bar{c}^{2}}
\end{array}\right]
$$

It should be noted that the form of $T(r)$ used in this work is equivalent to the one employed in [9].

\section{E. Implementation of Nonreflecting Boundary Condition and Interface}

The nonreflecting boundary condition and blade row interface presented in this work have been implemented into the finite-volume CFD solver G3D::Flow, which is developed and maintained at the Division of Fluid Dynamics at Chalmers University of Technology. In this work, G3D::Flow has been used to solve the nonlinear Euler equations in a coupled and conservative form. The fluid is modeled as an ideal gas and its properties are taken to be those of air. Inviscid fluxes over cell faces that arise from the finite-volume discretization are estimated using a low-dissipation, third-order accurate upwind scheme based on flux-vector splitting [15]. Temporal discretization for the unsteady computations performed in this work is further achieved with the Harmonic Balance method developed by Hall et al. [16]. A three-stage, second-order accurate Runge-Kutta cycle [15] is used to advance the discretized system in pseudo-time using local time-stepping until convergence is reached.

In G3D::Flow, two layers of ghost cells are used to calculate fluxes over a boundary or side of an interface. The aim of the nonreflecting implementation is to update these ghost cell values in such a way that the interior solution is driven towards a state that satisfies either Eq. (31) for a boundary, or Eq. (32) for an interface. In addition to this, the ghost cells must be updated so that the proper mean state is reached. For an inlet boundary, this should be done so that the mean flow matches a specified stagnation temperature, stagnation pressure, and flow direction, whereas for an outlet, a specified static pressure should be met. For an interface on the other hand, the aim should be to conserve the average flux of mass, momentum and energy over the interface.

\section{Nonzero Frequency and/or Azimuthal Wavenumber}

The first step in the construction of a nonreflecting boundary or interface is to sample the solution close to a boundary/interface to obtain a set of time-azimuthal Fourier coefficients. In G3D::Flow, this sampling requires the mesh close to the boundary to be structured into bands of constant radius, and will thus result in a set of Fourier coefficients for each cell-band. Let one of these Fourier coefficients be denoted $\hat{q}_{\mathrm{c}}\left(x_{\mathrm{c}}, r\right)$ to emphasize that it was sampled in a cell layer situated at axial location $x_{\mathrm{c}}$, radial location $r$, and inside the currently considered blade row. For each side of an interface, a set of Fourier coefficients representing the solution on the other side of the interface is also defined. This is done in two steps. First, the time-azimuthal Fourier coefficients sampled on the other side of the interface are interpolated to the radial location of the cell-bands in the currently considered blade row using area-weighted averaging. After this, each time-azimuthal Fourier coefficient sampled in the currently considered blade row is matched with an interpolated Fourier coefficient based on the condition defined in Section III.A. Let the time-azimuthal Fourier coefficient that matches $\hat{q}_{\mathrm{c}}\left(x_{\mathrm{c}}, r\right)$ be denoted $\hat{q}_{\mathrm{o}}\left(x_{\mathrm{o}}, r\right)$. As was explained in Section III.A a matching time-azimuthal Fourier coefficient from the other side of the interface may not always exist if a different number of temporal and azimuthal harmonics are used in the simulation. In these cases, $\hat{q}_{\mathrm{o}}\left(x_{\mathrm{o}}, r\right)$ is simply set to zero. For a boundary on the other hand, $\hat{q}_{\mathrm{o}}\left(x_{\mathrm{o}}, r\right)$ is always defined to be zero. This is done so that the algorithm used to construct a nonreflecting ghost cell state for an interface also can be used for a boundary.

For turbomachinery applications, G3D::Flow solves the governing equations formulated in Cartesian coordinates and the relative frame of reference. As such, the solution must first be converted to cylindrical coordinates and the absolute frame of reference before the time-azimuthal Fourier coefficients described in Section III.A can be computed. The nonreflecting analysis described hereinafter is also performed in the absolute frame of reference. This implies that both the absolute blade passing frequency in Eq. (5) and the absolute tangential velocity are used to compute 
the eigenvalues/eigenvectors. The absolute frame of reference is mainly chosen to make it convenient to match the samplings obtained on different sides of a blade row interface together. It should however be emphasized that the nonreflecting analysis presented in this paper would give equivalent results if the relative blade passing frequency and relative tangential velocity were used. This is first of all due to the fact that the factor $\left(\tilde{\omega}-k_{z} \bar{v}_{\theta}\right)$ gives the same results in both cases. Secondly, the sampling of the flow described in Section III.A gives the same time-azimuthal Fourier coefficients (except for $\hat{q}_{0,0, c}$ ) independent of whether the solution was converted to the absolute frame of reference or not before the sampling was performed.

Once the sampling of the flow has been completed as described above, a new set of Fourier coefficients $\hat{q}_{\text {nre }}\left(x_{l}, r\right)$ that define a nonreflecting exterior state for a given ghost cell layer $(l=1,2)$ and cell-band are computed according to Algorithm 1 It should be noted that the mean state $\bar{q}(r)$ that is passed to the algorithm is defined as follows for an interface

$$
\bar{q}(r)=\frac{\operatorname{Re}\left(\hat{q}_{0,0, \mathrm{c}}\left(x_{\mathrm{c}}, r\right)\right)+\operatorname{Re}\left(\hat{q}_{0,0, \mathrm{o}}\left(x_{\mathrm{o}}, r\right)\right)}{2}
$$

For a boundary on the other hand, the interior solution is simply chosen as the mean state. Algorithm 1 now works as follows. In the first steps, it calculates the modified frequency and all the axial wavenumbers. The parameter $\varepsilon$ used to calculate the modified frequency was taken to be $10^{-3} \bar{c} / P$, where $P=2 \pi r / N_{c}$. This value is similar to the one suggested by Frey et al. [8]. After the frequency and all the axial wavenumbers have been computed, the algorithm checks whether the boundary is an inlet or outlet by comparing the mean axial velocity to the axial component of the boundary normal $(n)$. If the boundary is an inlet, the algorithm proceeds by checking whether the second acoustic wave represents an incoming wave. If this is the case, it performs a sorting step to ensure that the first four waves will be incoming, and the fifth one outgoing. The reason for why it is sufficient to only swap the axial wavenumbers of the acoustic waves in order to sort them is that the eigenvectors in Eq. (19) have been defined as functions of $k_{x, 4}$ and $k_{x, 5}$. The next step in the algorithm checks whether the convected waves are resolved with at least $N$ points per wavelength by using the length of the cell layer closest to the interface $(\Delta x)$ as a reference. If this is not the case, the convected waves will be neglected in the analysis. This check has been introduced to ensure that high frequency waves not are included in the analysis. This is believed to be important since the axial wavenumbers of the convected waves can grow without bound when $\bar{v}_{x} \rightarrow 0$, which could be problematic when the modal amplitudes of the convected waves are phase extrapolated in subsequent steps of the algorithm. If the convected waves are included in the analysis, their modal amplitudes are calculated from the following expression

$$
\alpha_{\mathrm{o}}^{*}(x, r)=T^{-1}(r) \hat{q}_{\mathrm{o}}(x, r)
$$

In the next step of the algorithm the modal amplitude of the incoming acoustic wave is calculated from the above relation. The other acoustic wave is on the other hand obtained from the the interior solution

$$
\alpha_{\mathrm{c}}^{*}(x, r)=T^{-1}(r) \hat{q}_{\mathrm{c}}(x, r)
$$

In the next step of the algorithm a new vector $\alpha_{\text {nre }}^{*}$ is defined to contain the incoming modal amplitudes from the exterior and the outgoing modal amplitudes from the currently considered blade row. Note also that since $\alpha_{5, \mathrm{c}}^{*}$ has been calculated from the solution in the currently considered blade row, it is phase extrapolated to the position of the ghost cell layer before it is added to $\alpha_{\text {nre }}^{*}$. Phase extrapolation has also been used by e.g. Robens et al. [17]. The procedure used for an outlet is almost equivalent to the one used for the inlet, with the difference that there will be four outgoing waves and one incoming wave. In the final step of the algorithm, a new time-azimuthal Fourier coefficient that only contains incoming waves from the exterior, plus outgoing waves from the currently considered blade row, is calculated as follows

$$
\hat{q}_{\text {nre }}\left(x_{l}, r\right)=T(r) \alpha_{\text {nre }}^{*}\left(x_{l}, r\right)
$$

The procedure described above is repeated for all combinations of frequencies and azimuthal wave numbers (except $\omega=k_{z}=0$ ) that are included in the analysis. Once this has been done, a flow perturbation $q_{\mathrm{bc}}^{\prime}$ which prescribes a nonreflecting exterior state can be computed in the ghost cells by inserting $\hat{q}_{\text {nre }}$ into Eq. (3). This perturbation must then be combined with an updated mean flow before the flux over the boundary faces can be computed. The calculation of the mean state is outlined next. 


\section{Mean Flow}

One of the main reasons for applying a nonreflecting boundary condition to a turbomachinery calculation is that it enables the boundaries to be placed closer to the blades. When boundary conditions are to be specified on the other hand, the user will most likely only know the ambient conditions far upstream/downstream of the turbomachine. In order to relate the known ambient conditions with the flow close to the blades, mixed-out flow variables should be calculated from the non-uniform solution at the inlet and outlet boundaries [18]. Mixed-out variables have the property that they will be equal to the uniform state that the flow will attain once all non-uniformities in the flow have been

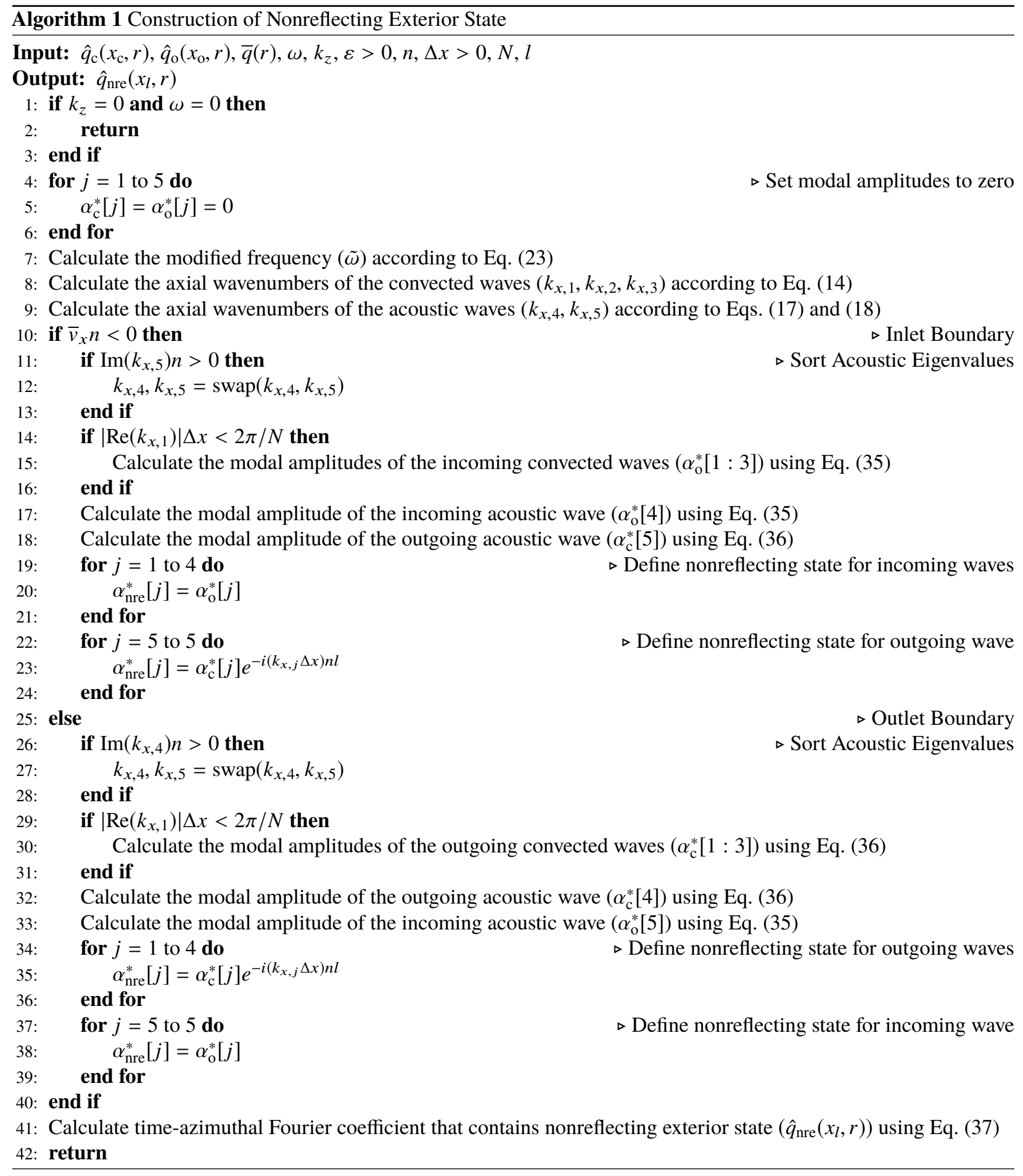


mixed out [18]. In his report on the UNSFLO code [18], Giles gives a detailed description of how these mixed-out variables can be used to impose a desired stagnation state at inlet, or static pressure at outlet. In addition to this, he also describes how the mixed-out variables can be used to construct a blade row interface which is conservative. These methods described by Giles are all based on calculating the difference between the mixed-out variables and the exterior state. For a boundary, the exterior state will be the user-imposed boundary condition, and for an interface, it will be the mixed-out variables obtained on the other side of the interface. This delta is then converted into 1D characteristic variables. Those characteristic variables that propagate into the domain are used to calculate a correction of the primitive variables, whereas the outwards propagating characteristics are discarded. This correction should then be applied to the time+azimuthal average state at the boundary/interface in order to obtain a new average state for the next iteration. Currently, the calculation of mixed-out variables has been implemented into G3D::Flow, but the construction of the correction remains. Therefore, a simpler method is used in this work for prescribing boundary conditions and matching the flow at a blade row interface. In this approach, a time-azimuthal average is calculated for all radial positions along the boundary. This average state is then extrapolated to the positions of the ghost cells and combined with the exterior state to obtain a new mean state $\bar{q}_{\mathrm{gc}}$. The procedure used for each type of boundary and interface is described below.

For an inlet boundary, the velocity in the ghost cells first calculated as

$$
\mathbf{u}_{\mathrm{gc}}=\frac{\mathbf{a}_{\mathrm{bc}}}{\left\|\mathbf{a}_{\mathrm{bc}}\right\|}\left\|\mathbf{u}_{\mathrm{e}}\right\|
$$

Here, subscript e and bc respectively denote the extrapolated state and the user defined state, and the vector a defines the flow direction. Once a velocity has been calculated, the static temperature in the ghost cells can be obtained from the user-defined stagnation temperature as

$$
T_{\mathrm{gc}}=T_{0, \mathrm{bc}}-\frac{\left\|\mathbf{u}_{\mathrm{gc}}\right\|^{2}}{2 C_{p}}
$$

The static pressure in the ghost cells is further calculated from the user-defined stagnation pressure and the newly computed temperature as

$$
p_{\mathrm{gc}}=P_{0, \mathrm{bc}}\left(\frac{T_{\mathrm{gc}}}{T_{0, \mathrm{bc}}}\right)^{\frac{\gamma}{\gamma-1}}
$$

In a final step the density in the ghost cells is calculated from the ideal gas equation of state to obtain a complete description of $\bar{q}_{\mathrm{bc}}$.

For an outlet, the specification of the ghost cell state is simpler. In this case, the static pressure in the extrapolated state is simply substituted with the specified static pressure in order to define an average state for the ghost cells.

The matching of the flow at a blade row interface starts by converting the extrapolated average state and the exterior state into 1D characteristic variables. Once this has been done, a new set of characteristic variables which contains the outgoing ones obtained from the extrapolated state and the incoming ones taken from the exterior state is assembled. This new set of characteristic variables is then converted back into primitive variables to obtain a new mean flow state in the ghost cells.

\section{Stabilization Strategy}

The perturbation state $q_{\mathrm{gc}}^{\prime}$ and the average state $\bar{q}_{\mathrm{gc}}$ defined in the previous sections may be combined to obtain a ghost cell state $q_{\mathrm{gc}}$ that is nonreflecting, and that defines the correct mean flow

$$
q_{\mathrm{gc}}=\bar{q}_{\mathrm{gc}}+q_{\mathrm{gc}}^{\prime}
$$

This state can now theoretically be used for calculating the flux over the boundary/interface. Unfortunately, this direct approach has been reported by several authors to be numerically unstable when an explicit pseudo-time integration strategy is used to converge the residual [4, 7, 9]. To overcome this issue, Giles originally proposed to reformulate Eq. (31) in terms of 1D characteristics [7]. This approach has also been adopted by other authors with good results [4, 9]. The authors of the present paper have however decided to stay with the construction of a nonreflecting ghost cell state described in Algorithm 1] There were two main motivations for this choice. First, it was found that the construction of a nonreflecting ghost cell state became significantly more complicated when the 1D characteristics approach was employed. Secondly, the authors wanted to find a stable implementation which also preserves the simple and straightforward approach defined in Algorithm 1 . 
To date, a simple approach for stabilizing the pseudo-time evolution problem has been found. In this approach, a new ghost cell state $\tilde{q}_{\mathrm{gc}}$ is computed in every iteration before the flux is calculated. This ghost cell state is obtained by locally extrapolating the interior solution to the position of the ghost cells, and then match the extrapolated state together with $q_{\mathrm{gc}}$ using 1D characteristics theory. The original ghost cell state $q_{\mathrm{gc}}$ is also only updated every $M^{\text {th }}$ iteration. In between the updates, the boundary/interface will thereby behave as a nonreflecting boundary condition based on local, 1D characteristics. This type of boundary condition is known to be well-posed for pseudo-time integration solvers [7]. The fact that the reference exterior state will be $q_{\mathrm{gc}}$ in between the updates also ensures that the interior solution will be driven towards a nonreflecting state which either satisfies Eq. (31) for a boundary, or Eq. (32) for an interface. The number of iterations between the updates must be chosen large enough to ensure that the interior solution has time to adapt to $q_{\mathrm{bc}}$. In this work, it was found that $M$ had to be in the order of a thousand iterations. If acceleration techniques, such as multigrid or implicit residual smoothing were to be applied, it is however believed that $M$ could be greatly reduced, since the interior solution would adapt faster to $q_{\mathrm{bc}}$. An extensive discussion on the stability and accuracy aspects of the nonreflecting formulation presented in this work can be found in [19].

\section{Results}

\section{A. Two-dimensional Wave Propagation}

A set of two-dimensional validation cases were defined in order to verify the implementation of the nonreflecting boundary condition and the nonreflecting blade row interface. The computational domain used for these validation cases is depicted in Fig. 1. To begin with, however, only the stationary sub domain to the right in this figure was included in the simulations. For these cases, the upstream and downstream boundary of the second sub domain were set to be a stagnation inlet and pressure outlet respectively. When the moving sub domain in Fig. 11 later was included in the simulations, the leftmost boundary was instead set to be a stagnation inlet and a blade row interface was used to connect the two sub domains. Translational periodicity was applied at the periodic boundaries in all cases. For each of the validation cases, a specific wave was prescribed at one of the boundaries. This wave was then allowed to propagate through the domain, cross the blade row interface (in cases where it was included), and finally impinge on the other boundary. The implementation could then be verified by comparing the numerically computed wave with the analytical solution defined by Eq. 10 .

A specification of all the waves which were investigated in this work is provided in Table 2 . Note that in this table, a and $k_{z}$ denote the direction of the mean flow in the absolute frame of reference and the y-component of the wavenumber respectively. The reason for the latter choice of nomenclature is that the circumferential direction will correspond to the $y$-direction when the equations defined in the previous sections are applied to two dimensional problems. The same computational mesh was used for all simulations defined in Table 2, and the mesh resolution was set to ensure that each wave was resolved with at least 30 points per wavelength along each coordinate axis.

All simulations performed in this work were converged to machine precision. Solution data was then extracted on an axial line between the inlet and the outlet and plotted against the corresponding analytical solution. The results obtained are presented in Fig. 2 8 The agreement between the analytical and the numerical solution can be seen to be is excellent for all cases except the one presented in Fig. 7. This specific case represents the upstream propagating acoustic wave, whose amplitude can be seen to be slightly under-predicted in the numerical solution. This discrepancy is believed to stem from numerical dissipation. It is also interesting to note from Fig. 4 and Fig. 6 that the blade row interface does not seem to have any effect on the wave passing by. This is an important result, since it shows that the wave is appropriately Doppler-shifted when it is passed over the interface.

Table 1 Size of computational domain depicted in Fig. 1 .

\begin{tabular}{cccc}
\hline$d_{1}$ & $d_{2}$ & $d_{3}$ & $d_{4}$ \\
$5 \mathrm{~m}$ & $1 \mathrm{~m}$ & $5 \mathrm{~m}$ & $1 \mathrm{~m}$ \\
\hline
\end{tabular}




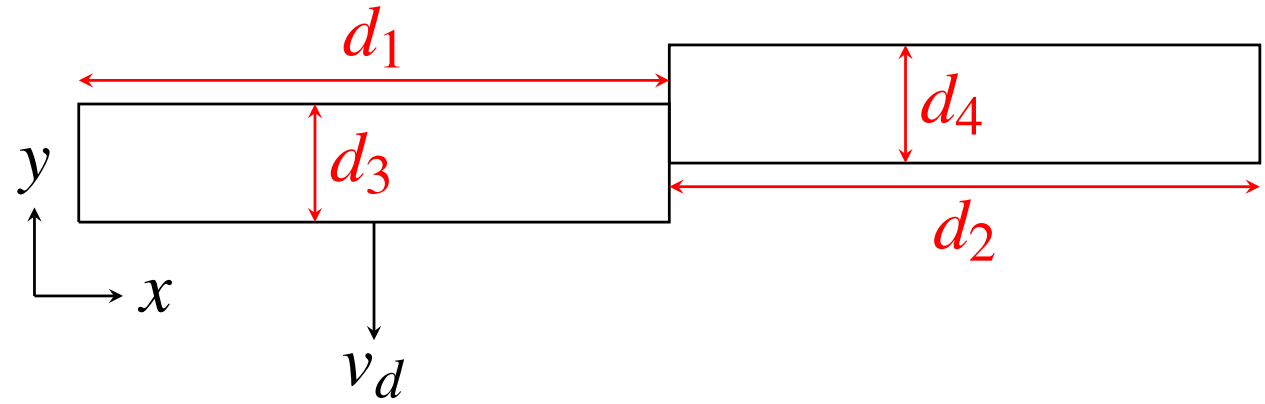

Fig. 1 Schematic view of the computational domain used to verify the implementation. The sizes shown are listed in Table 1 .

Table 2 Simulation settings used for the different validation cases.

\begin{tabular}{|c|c|c|c|c|c|c|c|c|}
\hline & $\begin{array}{c}T_{0, \text { in }} \\
\mathrm{K}\end{array}$ & $\begin{array}{c}P_{0, \text { in }} \\
\mathrm{Pa}\end{array}$ & $\mathbf{a}$ & $\begin{array}{c}p_{\text {out }} \\
\mathrm{Pa}\end{array}$ & $\begin{array}{c}\omega \\
\operatorname{rad~s}^{-1}\end{array}$ & $\begin{array}{c}k_{z} \\
\operatorname{rad~m}^{-1}\end{array}$ & $\begin{array}{c}k_{x, j} \\
\operatorname{rad~m}^{-1}\end{array}$ & $\begin{array}{c}v_{d} \\
\mathrm{~m} \mathrm{~s}^{-1}\end{array}$ \\
\hline Steady & & & & & & & & \\
\hline $\begin{array}{c}\text { Vorticity Wave } \\
\text { Propagating }\end{array}$ & 104.73 & 47765.58 & $(5,1)$ & 40000 & 0 & $2 \pi$ & -1.2566 & - \\
\hline $\begin{array}{c}\text { Vorticity Wave } \\
\text { Propagating } \\
\text { Vorticity Wave }\end{array}$ & 106.32 & 50356.95 & $(5,-3)$ & 40000 & -502.65 & $2 \pi$ & -1.2566 & - \\
\hline $\begin{array}{l}\text { Including Interface } \\
\text { Downstream Traveling }\end{array}$ & 106.32 & 50356.95 & $(5,-3)$ & 40000 & -502.65 & $2 \pi$ & -1.2566 & -80 \\
\hline $\begin{array}{c}\text { Acoustic Wave } \\
\text { Downstream Traveling }\end{array}$ & 106.32 & 50356.95 & $(5,-3)$ & 40000 & -1005.31 & $-2 \pi$ & -1.0743 & - \\
\hline $\begin{array}{l}\text { Acoustic Wave } \\
+ \text { Interface }\end{array}$ & 106.32 & 50356.95 & $(5,-3)$ & 40000 & -1005.31 & $-2 \pi$ & -1.0743 & -80 \\
\hline $\begin{array}{c}\text { Upstream Traveling } \\
\text { Acoustic Wave }\end{array}$ & 106.32 & 50356.95 & $(5,-3)$ & 40000 & -772.83 & $-2 \pi$ & 6.3070 & - \\
\hline $\begin{array}{c}\text { Downstream Traveling } \\
\text { Acoustic Wave }\end{array}$ & & & & & & & & \\
\hline+ Cut-Off & 105.49 & 48997.24 & $(5,-2.2)$ & 40000 & -804.25 & $-2 \pi$ & $3.60-0.85 i$ & - \\
\hline
\end{tabular}




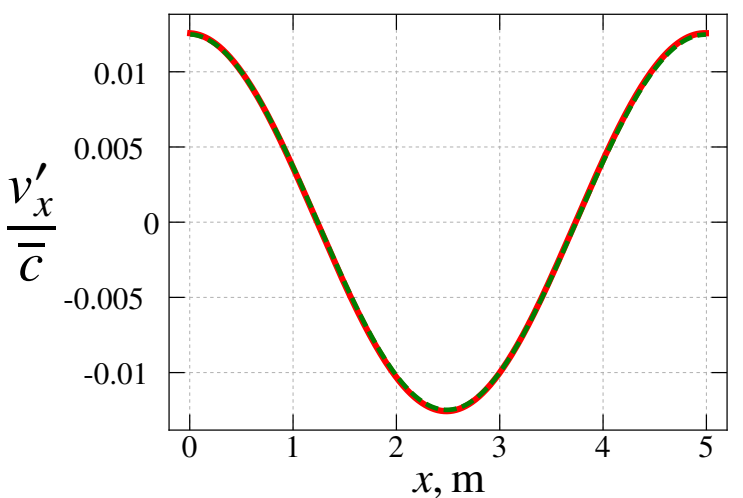

(a) Axial component of velocity perturbation normalized (b) Tangential component of velocity perturbation normalby speed of sound.

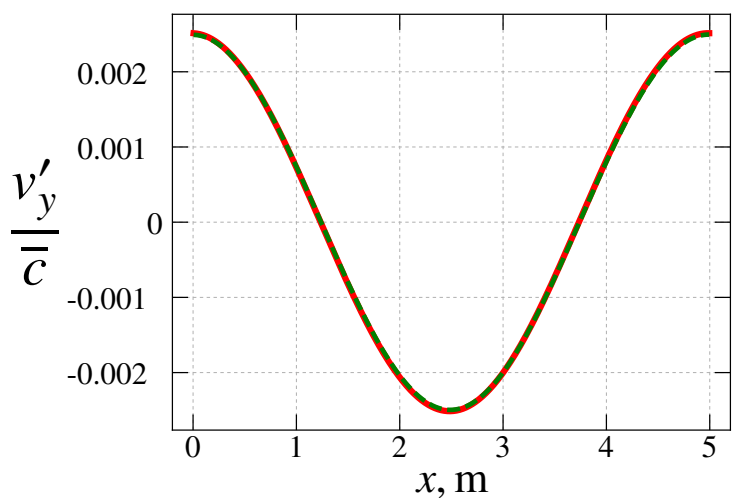

ized by speed of sound.

Fig. 2 Normalized velocity perturbations for the steady vorticity wave (Analytical solution: - , Numerical solution: ---- ).

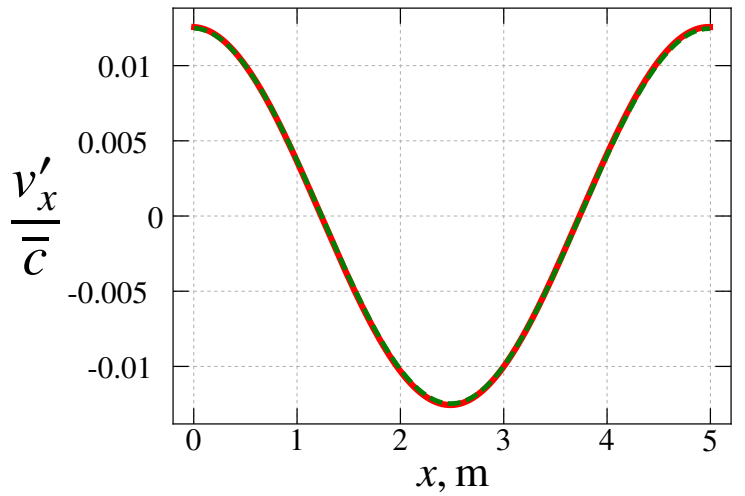

(a) Axial component of velocity perturbation normalized (b) Tangential component of velocity perturbation normalby speed of sound.

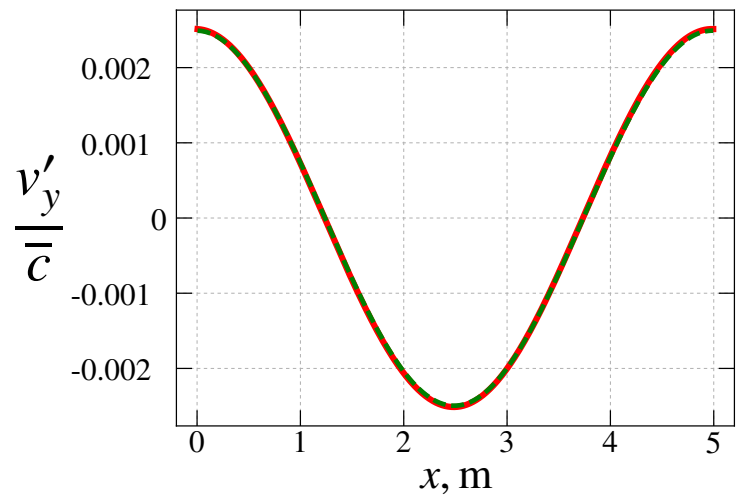

ized by speed of sound.

Fig. 3 Normalized velocity perturbations for the propagating vorticity wave (Analytical solution: - , Numerical solution: - - - - ). 


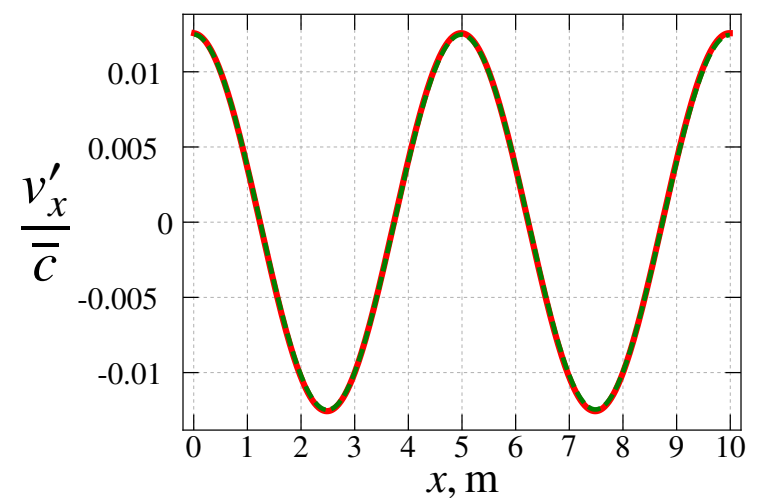

(a) Axial component of velocity perturbation normalized (b) Tangential component of velocity perturbation normalby speed of sound.

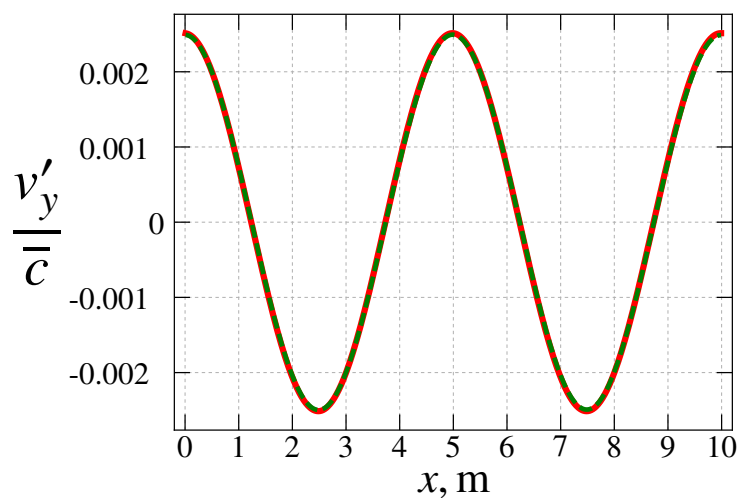

ized by speed of sound.

Fig. 4 Normalized velocity perturbations for the propagating vorticity wave when an interface is included at $x=5 \mathrm{~m}$ (Analytical solution: $\longrightarrow$, Numerical solution: - - - - ).

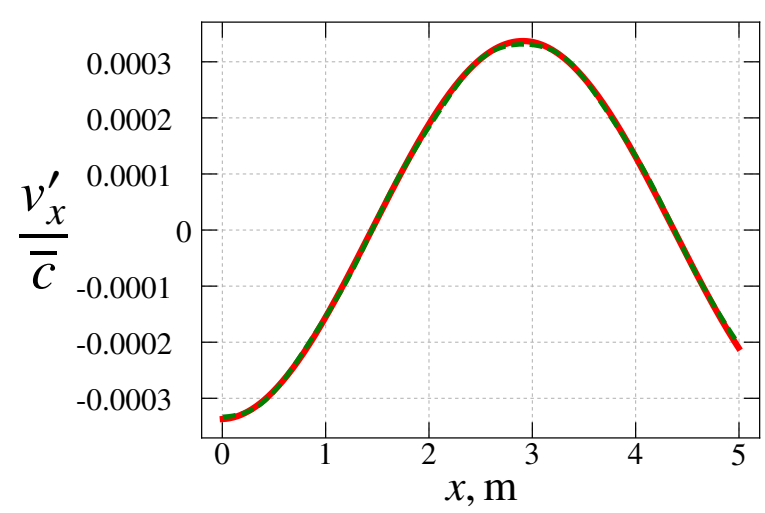

(a) Axial component of velocity perturbation normalized by speed of sound.

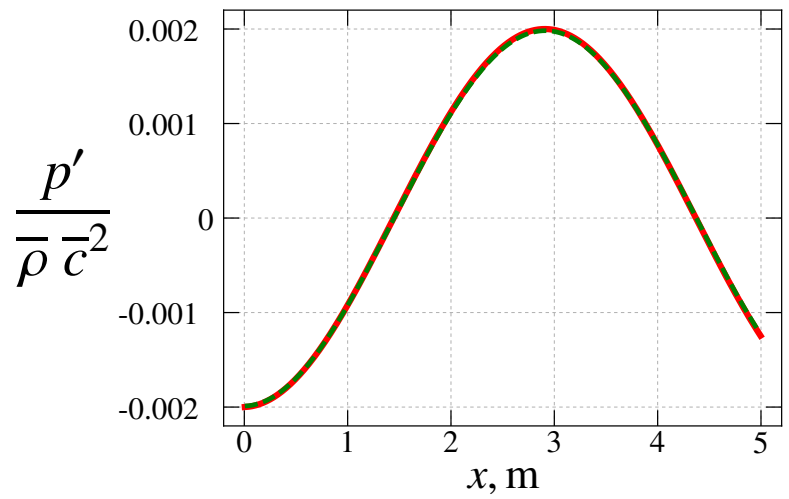

(b) Pressure perturbation normalized by density and speed of sound.

Fig. 5 Normalized velocity and pressure perturbations for the downstream traveling acoustic wave (Analytical solution: - Numerical solution: - - - - ). 

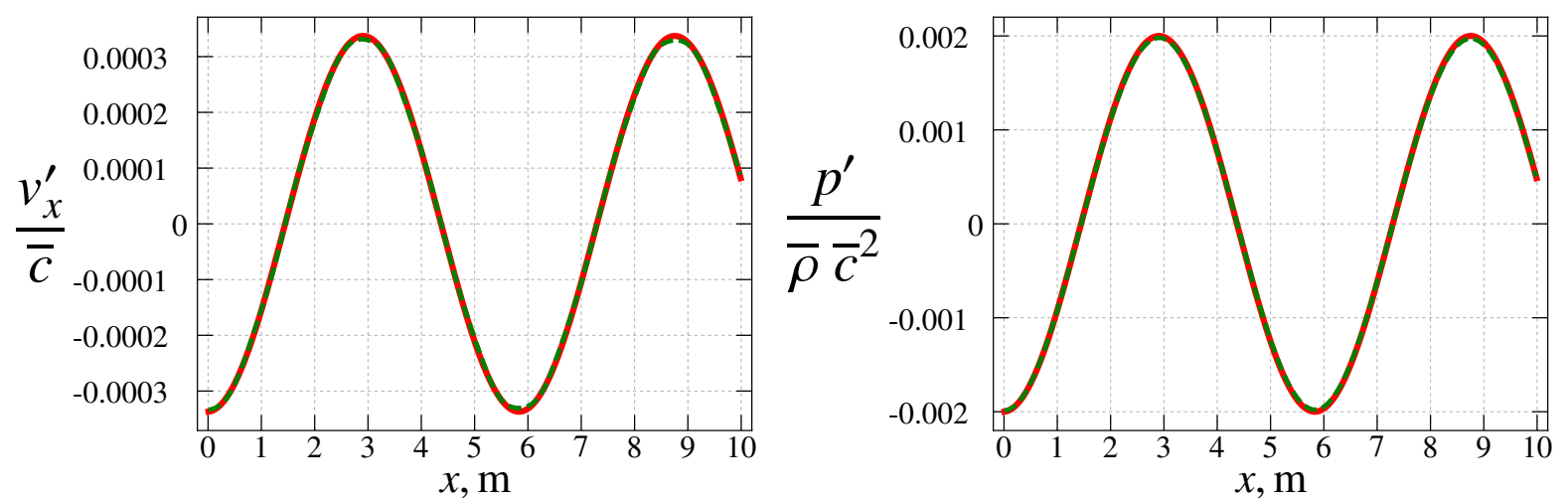

(a) Axial component of velocity perturbation normalized (b) Pressure perturbation normalized by density and speed by speed of sound. of sound.

Fig. 6 Normalized velocity and pressure perturbations for the downstream traveling acoustic wave when an interface is included at $x=5 \mathrm{~m}$ (Analytical solution:

- , Numerical solution: ---- ).
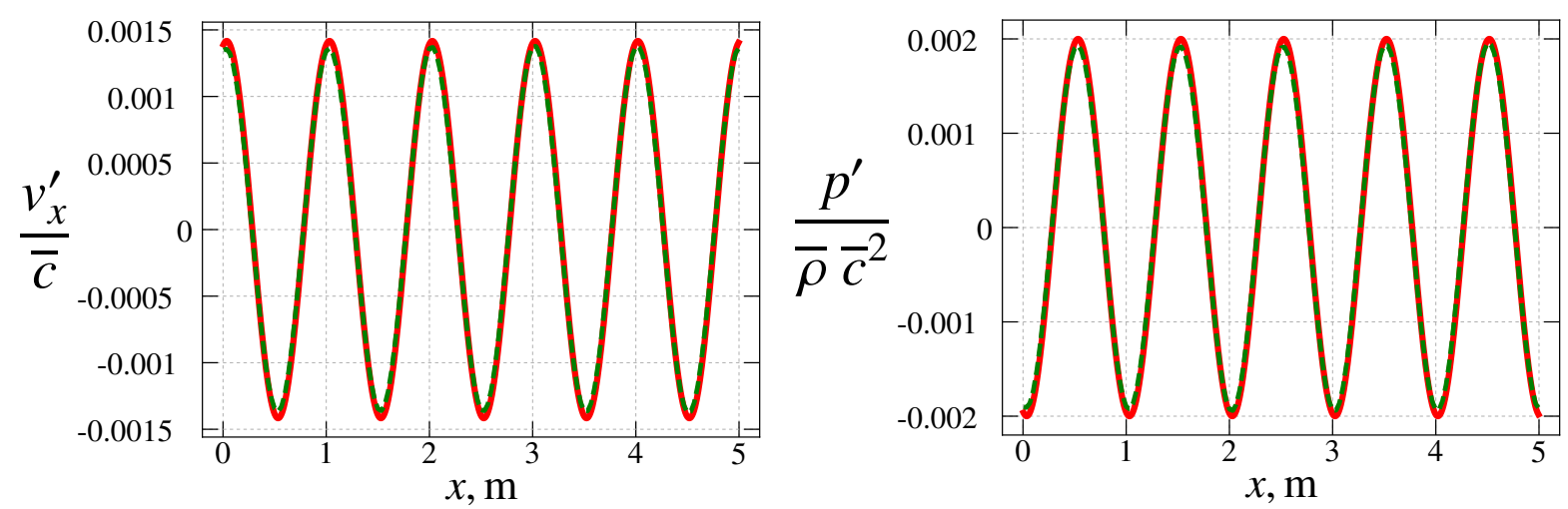

(a) Axial component of velocity perturbation normalized (b) Pressure perturbation normalized by density and speed by speed of sound. of sound.

Fig. 7 Normalized velocity and pressure perturbations for the upstream traveling acoustic wave (Analytical solution: - Numerical solution: - - - - ). 


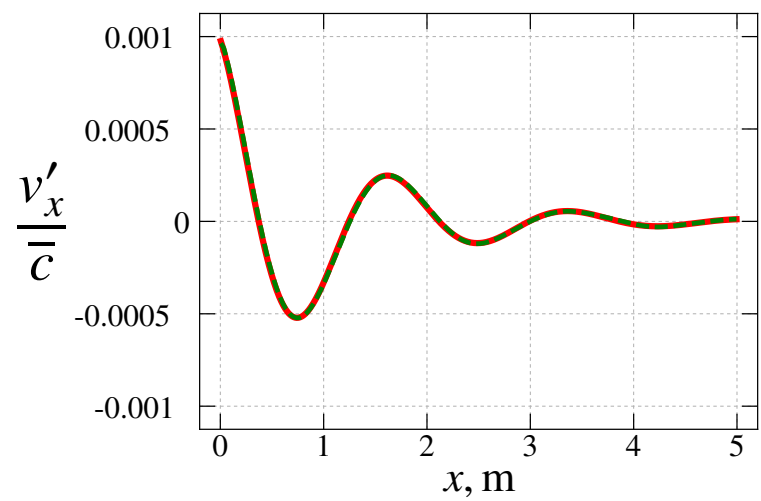

(a) Axial component of velocity perturbation normalized by speed of sound.

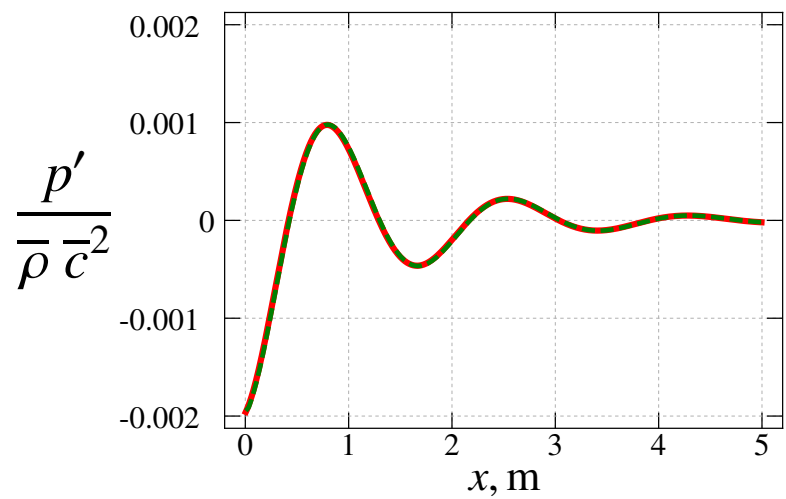

(b) Pressure perturbation normalized by density and speed of sound.

Fig. 8 Normalized velocity and pressure perturbations for the downstream traveling acoustic wave which is cut-off (Analytical solution: - , Numerical solution: $=--=$ ).

\section{Conclusion}

In this paper, a quasi-three-dimensional nonreflecting formulation applicable to turbomachinery boundaries and blade row interfaces has been presented. The formulation is based on the exact, nonreflecting boundary condition for a single frequency and azimuthal wave number developed by Giles [1,7], which makes the formulation non-local in both space and time. Special effort was put into the derivation of the formulation to ensure that it can be applied in a wide variety of situations without modification. As a result of this effort, a formulation was obtained which works both for steady state and unsteady simulations. The formulation also avoids problems associated with acoustic resonance by including the regularization proposed by Frey et al. [8].

A detailed description of how the chosen formulation was implemented into an explicit finite-volume solver was also presented. With the chosen implementation strategy, convergence to machine-zero is obtained for a set of two-dimensional wave-propagation problems. This shows that although the implemented algorithm is sub-optimal from a computational-efficiency point of view, it yields an algorithm that, at least for the cases considered, is numerically stable.

The two-dimensional wave-propagation problems were also used to verify the implementation. In each of these problems, a particular type of wave which is supported by the nonreflecting formulation was excited at the upstream or downstream boundary, allowed to propagate through the domain, and then finally impinge on the other boundary. Very good agreement between numerical and analytical results were demonstrated in all the cases. In particular, there were no signs of reflections or distortions of the waves as they propagated through the domain. This shows that the presented formulation both has been correctly implemented, and that it represents physically correct waves.

\section{Acknowledgments}

This work is financially supported by the E.U. under the ULTIMATEFProject funded by the European Commission within the Horizon 2020 Programme (2014-2020) under the Grant Agreement No 633436.

\section{References}

[1] Giles, M. B., "Nonreflecting Boundary Conditions for Euler Equation Calculations," AIAA Journal, Vol. 28, No. 12, 1990, pp. 2050-2058.

[2] Saxer, A. P., and Giles, M. B., "Quasi-Three-Dimensional Nonreflecting Boundary Conditions for Euler Equations Calculations," Journal of Propulsion and Power, Vol. 9, No. 2, 1993, pp. 263-271.

[3] Frey, C., Ashcroft, G., Kersken, H.-P., and Weckmüller, C., "Advanced Numerical Methods for the Prediction of Tonal

\footnotetext{
*Ultra Low emission Technology Innovations for Mid-century Aircraft Turbine Engines
} 
Noise in Turbomachinery — Part II: Time-Linearized Methods," Journal of Turbomachinery, Vol. 136, No. 2, 2014, pp. 021003-1-021003-10.

[4] Frey, C., Ashcroft, G., and Kersken, H.-P., "Simulations of Unsteady Blade Row Interactions Using Linear and Non-Linear Frequency Domain Methods,” ASME Turbo Expo 2015: Turbine Technical Conference and Exposition, GT2015-43453, 2015.

[5] Ekici, K., and Hall, K. C., "Nonlinear Analysis of Unsteady Flows in Multistage Turbomachines Using Harmonic Balance," AIAA Journal, Vol. 45, No. 5, 2007, pp. 1047-1057.

[6] Engquist, B., and Majda, A., "Absorbing Boundary Conditions for the Numerical Simulation of Waves," Mathematics of Computation, Vol. 31, No. 139, 1977, pp. 629-651.

[7] Giles, M. B., "Non-Reflecting Boundary Conditions for the Euler Equations," CFDL-TR-88-1, 1988.

[8] Frey, C., and Kersken, H.-P., "On the Regularisation of Non-Reflecting Boundary Conditions near Acoustic Resonance," VII European Congress on Computational Methods in Applied Sciences and Engineering, 2016.

[9] Kersken, H.-P., Ashcroft, G., Frey, C., Wolfrum, N., and Korte, D., "Nonreflecting Boundary Conditions for Aeroelastic Analysis in Time and Frequency Domain 3D RANS Solvers," ASME Turbo Expo 2014: Turbine Technical Conference and Exposition, GT2014-25499, 2014.

[10] Lindblad, D., Montero Villar, G., Andersson, N., and Wukie, N. A., "Implementation of a Quasi-Three-Dimensional Nonreflecting Blade Row Interface for Steady and Unsteady Analysis of Axial Turbomachines," 2018 AIAA Aviation and Aeronautics Forum and Exposition, 2018.

[11] Olausson, M., "Turbomachinery Aeroacoustic Calculations using Nonlinear Methods,” Ph.D. thesis, Division of Fluid Dynamics, Department of Applied Mechanics, Chalmers University of Technology, Gothenburg, Sweden, 2011.

[12] Tyler, J. M., and Sofrin, T. G., “Axial Flow Compressor Noise Studies,” SAE Technical Paper, 620532, 1962.

[13] Moinier, P., Giles, M. B., and Coupland, J., "Three-Dimensional Nonreflecting Boundary Conditions for Swirling Flow in Turbomachinery," Journal of Propulsion and Power, Vol. 23, No. 5, 2007, pp. 981-986.

[14] Moinier, P., and Giles, M. B., "Eigenmode Analysis for Turbomachinery Applications," Journal of Propulsion and Power, Vol. 21, No. 6, 2005, pp. 973-978.

[15] Andersson, N., "A Study of Subsonic Turbulent Jets and Their Radiated Sound Using Large-Eddy Simulation," Ph.D. thesis, Division of Fluid Dynamics, Department of Applied Mechanics, Chalmers University of Technology, Gothenburg, Sweden, 2005.

[16] Hall, K. C., Jeffrey, P. T., and Clark, W. S., "Computation of Unsteady Nonlinear Flows in Cascades Using a Harmonic Balance Technique,” AIAA Journal, Vol. 40, No. 5, 2002, pp. 879-886.

[17] Robens, S., Jeschke, P., Frey, C., Kügeler, E., Bosco, A., and Breuer, T., "Adaptation of Giles Non-Local Non-Reflecting Boundary Conditions for a Cell-centered Solver for Turbomachinery Applications," ASME Turbo Expo 2013: Turbine Technical Conference and Exposition, GT2013-94957, 2013.

[18] Giles, M. B., “UNSFLO: A Numerical Method For The Calculation Of Unsteady Flow In Turbomachinery,” GTL Report 205, 1991.

[19] Wukie, N. A., Orkwis, P. D., Lindblad, D., and Andersson, N., "Nonreflecting boundary conditions for the Euler equations in a discontinuous Galerkin discretization,” 2019 AIAA Aerospace Sciences Meeting, 2019. 